(c) 2019 Universidad Nacional Autónoma de México, Facultad de Estudios Superiores Zaragoza.

Este es un artículo Open Access bajo la licencia CC BY-NC-ND (http://creativecommons.org/licenses/by-nc-nd/4.0/).

TIP Revista Especializada en Ciencias Químico-Biológicas, 22: 1-8, 2019.

DOI: $10.22201 /$ fesz.23958723e.2019.0.189

\title{
Evaluación in vitro del quitosano y aceites esenciales para el control de dos especies patógenas de Colletotrichum aisladas de aguacate (Persea americana Mill)
}

\author{
Mireya Esbeiddy Chávez-Magdaleno ${ }^{1}$, Porfirio Gutiérrez-Martínez ${ }^{1}$, \\ Beatriz Montaño-Leyva ${ }^{2}$ y Ramsés Ramón González-Estrada ${ }^{1 *}$ \\ ${ }^{1}$ Tecnológico Nacional de México/Instituto Tecnológico de Tepic, Laboratorio Integral \\ de Investigación en Alimentos, Av. Tecnológico \#2595, Tepic 63175, Nayarit, México. \\ ${ }^{2}$ Universidad de Sonora, Depto. de Investigación y Posgrado en Alimentos, \\ Hermosillo 83000, Sonora, México. E-mail: *ramgonzalez@ittepic.edu.mx
}

\begin{abstract}
RESUMEN
La actividad antifúngica del quitosano, aceites esenciales (canela y eucalipto) y su combinación fueron evaluadas contra dos patógenos Colletotrichum acutatum y Colletotrichum gloeosporioides aislados de aguacate (Persea americana Mill). Se utilizó quitosano a diferentes concentraciones ( $0.1,0.5$ y $1.0 \%)$. Las concentraciones de aceites esenciales evaluadas fueron $0.1,0.5$ y $2.0 \%$ (eucalipto y canela). El efecto de la combinación del quitosano con los aceites esenciales se evaluó utilizando las concentraciones más eficaces del quitosano $(0.1$ y $0.5 \%)$ y el aceite esencial de eucalipto y canela con el mayor efecto inhibitorio (2\%). Los tratamientos de quitosano y aceites esenciales cada uno por separado fueron parcialmente efectivos para evitar el desarrollo de las cepas evaluadas, inhibiendo el crecimiento micelial en un rango de 17 a $21 \%$ y 18 a 50\% respectivamente. Por lo contrario, el crecimiento micelial de ambas cepas fue totalmente reducido mediante la aplicación combinada del quitosano $(0.1 \%)$ con aceite esencial de canela $(2 \%)$, sin embargo; la combinación del quitosano con aceite esencial de eucalipto no fue efectiva. Hasta donde sabemos, esta investigación es la primera que informa sobre cepas de Colletotrichum resistentes al quitosano aisladas de frutos de aguacate.

Palabras Clave: antifúngico, esporas, biopolímero, extractos vegetales.
\end{abstract}

In vitro assessment of chitosan and essential oils for the control of two pathogen species of Colletotrichum isolated from avocado (Persea americana Mill)

\begin{abstract}
The antifungal activity of chitosan, essential oils (cinnamon and eucalyptus) and their combination were evaluated against two pathogens Colletotrichum acutatum and Colletotrichum gloeosporioides isolated from avocado (Persea americana Mill). Chitosan was used at different concentrations (0.1, 0.5 and $1.0 \%)$. The concentrations of essential oils evaluated were $0.1,0.5$ and $2.0 \%$ (eucalyptus and cinnamon). The effect of the combination of chitosan with the essential oils was evaluated using the most effective concentrations of chitosan $(0.1$ and $0.5 \%)$ and the essential oil with the highest inhibitory effect (2\%).The treatments of chitosan and essential oils were partially effective against fungal development by inhibiting mycelial growth in a range of 17 to $21 \%$ and 18 to $50 \%$, respectively. The mycelia growth of both strains was totally reduced by the application of chitosan $(0.1 \%)$ with cinnamon essential oil $(2 \%)$, however; the combination of chitosan with eucalyptus essential oil was not effective. To our knowledge this research is the first that reports chitosan resistant strains of Colletotrichum isolated from avocado fruits.
\end{abstract}

Key Words: antifungal, spores, biopolymer, vegetables extracts.

Nota: Artículo recibido el 28 de febrero del 2019 y aceptado el 5 de septiembre del 2019. 


\section{INTRODUCCIÓN}

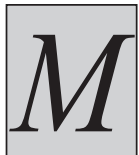

éxico, es el principal proveedor de aguacate para el mercado internacional, con una contribución del $45 \%$ del valor de las exportaciones mundiales (SAGARPA, 2017). En ambientes tropicales y subtropicales húmedos, la producción comercial del aguacate se considera una inversión de alto riesgo debido a las pérdidas causadas por enfermedades en pre y postcosecha. La antracnosis es la principal enfermedad del aguacate provocada por hongos del género Colletotrichum que pudre la fruta y en consecuencia el deterioro de la calidad afecta su comercialización (Trinidad-Ángel et al., 2017).Tradicionalmente, para el control de la infección por patógenos se han venido aplicando fungicidas químicos, sin embargo, los residuos químicos pueden ser peligrosos para los humanos y otros organismos (Kim, Kabir \& Jahan, 2017; Sarkhosh et al., 2017). En la actualidad, la demanda de productos agrícolas orgánicos va en aumento, por lo anterior, es necesario buscar tratamientos de fungicidas alternativos de contacto, que sean seguros y eficaces (Reganold \& Wachter, 2016). En este sentido, el quitosano es derivado de la quitina (polisacárido de origen animal) y es el principal constituyente del exoesqueleto de insectos y crustáceos. El quitosano es el derivado del $\mathrm{N}$-desacetilado de la quitina, que es un biopolímero de diferentes pesos moleculares (bajo, medio, alto), biodegradable, antifúngico y no tóxico para el humano y las plantas. Este biopolímero ha sido utilizado para el control de diversos patógenos como Rhizopus stolonifer, Penicillium expansum, Colletotrichum gloeosporioides aislados de diferentes frutas, como: guanábana, tomate, pera, mango, entre otros (Romanazzi, Feliziani, Baños \& Sivakumar, 2017). Se han reportado importantes cambios en la morfología de hongos expuestos al quitosano como: ruptura de la pared celular, desorganización de la membrana celular, distribución tanto anormal como cuerpos de inclusión no membranosos en el citoplasma, así como un engrosamiento de las paredes de las hifas dando lugar a deformaciones (Romanazzi, Feliziani, Baños \& Sivakumar, 2017). Por otro lado, los aceites esenciales son reconocidos como seguros y se ha reportado su eficacia para el control de patógenos de frutas y hortalizas (Prakash, Kedia, Mishra \& Dubey, 2015; España, Arboleda, Ribeiro, Abdelnur \& Guzman, 2017; Rabari Chudashama \& Thaker, 2018; Taghavi, Kim \& Rahemi, 2018). No obstante, la aplicación independiente del quitosano o aceites esenciales, en algunos casos no son efectivos contra los hongos. Por lo que, el uso combinado del quitosano con aceites esenciales puede ser una alternativa viable contra los patógenos que afectan a las frutas. El objetivo de esta investigación consistió en evaluar el efecto del quitosano y aceites esenciales (eucalipto y canela) de manera independiente y su combinación contra el desarrollo de C. acutatum y C. gloeosporioides in vitro.

\section{MATERIALES Y MÉTODOS \\ Material biológico}

Los hongos en estudio fueron previamente aislados e identificados de aguacates (Persea americana Mill) infectados. Las cepas de C. acutatum y $C$. gloeosporioides se mantuvieron en medio agar papa dextrosa (APD, SigmaAldrich, St. Louis, MO, EE. UU.) a $25{ }^{\circ} \mathrm{C}$ (Ramos-Guerrero et al., 2018).

\section{Preparación de soluciones Quitosano}

Las soluciones de quitosano se prepararon al $0.1,0.5$ y $1.0 \%$ de la siguiente manera: se realizaron soluciones disolviendo $0.1,0.5$ y $1.0 \mathrm{~g}$ de quitosano peso molecular medio $(\mathrm{cp}=590$, desacetilación al 75-85\%) (Sigma-Aldrich, St. Louis, MO, EE. UU.), en $100 \mathrm{~mL}$ de ácido acético al 2\% (v/v), se agitó por 24 horas a temperatura ambiente, las soluciones se ajustaron a $\mathrm{pH}$ de 5.5 agregando $\mathrm{NaOH}$ al $1 \mathrm{~N}$, se añadieron $100 \mu \mathrm{L}$ de Tween 80 (El Ghaouth, Arul, Grenier \& Asselin, 1992). Se esterilizaron las soluciones de quitosano por separado. Posteriormente, se preparó el medio APD de acuerdo a las instrucciones del proveedor, para mezclarlo con el quitosano a distintas concentraciones $(0.1,0.5$ y $1.0 \%)$; lo obtenido se vació en diferentes cajas Petri y una vez solidificado se procedió a inocular este medio con los hongos de estudio, seguido de la evaluación de su crecimiento. El tratamiento control consistió en cajas Petri con medio APD sin quitosano.

\section{Aceites esenciales de canela y eucalipto}

Los aceites esenciales de eucalipto (Pronaur ${ }^{\circledR}$, Guadalajara México, https://pronaur.negocio.site/) y canela (Pronaur $^{\circledR}$, Guadalajara México, https://pronaur.negocio.site/), se adquirieron en un centro de productos naturistas. Los aceites esenciales como parte de los tratamientos aplicados se prepararon de la siguiente manera: el medio de cultivo APD se esterilizó y cuando disminuyó su temperatura se mezcló con los aceites esenciales (canela y eucalipto) cada uno a las diferentes concentraciones evaluadas $(0.1,0.5$ y $2.0 \%$ ), una vez solidificados se utilizaron como medio de cultivo, inoculando los hongos de estudio. Por su parte el tratamiento control consisitió en un medio de cultivo APD sin aceites esenciales. Una vez determinada la concentración de aceite esencial con mayor actividad antifúngica, ésta se utilizó para la combinación con quitosano a bajas concentraciones. Con el fin de evaluar la efectividad de la combinación del quitosano con aceites esenciales se llevó a cabo el siguiente protocolo: se esterilizaron por separado el medio de cultivo APD y el quitosano, después se mezclaron, al disminuir la temperatura a $28{ }^{\circ} \mathrm{C}$ se incorporó cada aceite esencial al $2 \%$ y el quitosano a las diferentes concentraciones evaluadas ( 0.1 y $0.5 \%)$, una vez solidificado el medio de cultivo en la campana de flujo laminar, se inoculó con los hongos de estudio. El tratamiento 
control consistió en un medio de cultivo (APD) sin aceites esenciales ni quitosano.

\section{Análisis de efectividad in vitro \\ Evaluación de la inhibición del crecimiento micelial}

En el medio de cultivo APD y utilizando un sacabocados se tomó un disco de $7 \mathrm{~mm}$ de diámetro que contenía el micelio de cada uno de los hongos de estudio (con crecimiento activo de diez días), para inocularlos en el centro de las cajas de Petri que contenían los tratamientos descritos en el párrafo anterior, estas cajas fueron incubadas a $25^{\circ} \mathrm{C}$; el diámetro micelial se midió con un vernier $\left(\right.$ Truper $\left.^{\circledR}\right)$ cada 24 horas durante 10 días. Los resultados se reportaron como porcentaje de inhibición en comparación con el control aplicando la fórmula propuesta por Fagundes, Pérez-Gago, Monteiro \& Palou (2013).

\section{Evaluación de la inhibición de la germinación de esporas}

La efectividad de los tratamientos se evaluó de la siguiente manera: se cuadriculó el medio APD que contenían los tratamientos descritos, cada cuadro de $1 \mathrm{~cm} \times 1 \mathrm{~cm}$; y se preparó una suspensión de esporas empleando la metodología propuesta por González-Estrada, Calderón-Santoyo, RagazzoSánchez, Peyron \& Chalier (2018) a una concentración de $1 \times 10^{6}$ esporas $/ \mathrm{mL}$, posteriormente se tomaron alícuotas de $20 \mu \mathrm{L}$ para verterlas en los cuadros mencionados. Finalmente el medio APD inoculado se colocó en el microscopio para observar la germinación de las esporas. El conteo de la germinación de las esporas se realizó cada hora durante doce horas a partir de la inoculación, empleando un microscopio (Motic BA 300) a 40x en muestras de 200 esporas. Las esporas se consideraron germinadas cuando la longitud del tubo germinativo fue el doble del diámetro de la misma (Yao, Tian \& Wang, 2004). El control consistió en medio APD inoculado.

\section{Esporulación}

En la evaluación de la esporulación se utilizaron las mismas cajas Petri donde se midió el crecimiento micelial empleando el protocolo propuesto por Ramos-Guerrero et al. (2018). El procedimiento fue el siguiente: se agregaron $10 \mathrm{~mL}$ de agua destilada estéril a las cajas Petri y en consecuencia se raspó la superficie con ayuda de una varilla de vidrio estéril, posteriormente se filtró el contenido empleando una gasa para separar el micelio; enseguida se tomaron $100 \mu \mathrm{L}$ de la solución filtrada y se vertieron en la cámara de Neubauer $\left(\right.$ Loptik labor $\left.^{\circledR}\right)$. El conteo se llevó a cabo empleando un microscopio óptico a 40x. Los resultados se expresaron en número de esporas $/ \mathrm{mL}$.

\section{Análisis estadístico}

El análisis se realizó utilizando cinco cajas de Petri por tratamiento con tres repeticiones. Cada experimento se realizó por duplicado, utilizando un diseño de bloques completamente al azar. Los datos se sometieron a análisis de varianza (ANOVA) y se utilizó una prueba de Tukey $(\mathrm{P}<0.05)$ para la comparación de medias, empleando el paquete estadístico SAS versión 9.4.

\section{RESULTADOS Y DISCUSIÓN}

Efecto del quitosano sobre el crecimiento in vitro de $C$. acutatum y $C$. gloeosporioides

Los resultados del crecimiento micelial de los fitopatógenos se muestran en la Tabla I. Se observa una disminución del crecimiento micelial con respecto al control, con quitosano al $1.0 \%$ de tan solo un 17 y $21 \%$ de inhibición para C. acutatum y C. gloeosporioides, respectivamente. La germinación (Tabla I) fue totalmente inhibida con las diferentes concentraciones de quitosano evaluadas. $\mathrm{Se}$ observó diferencia significativa $(\mathrm{P}<0.05)$ entre los tratamientos para todos los parámetros evaluados (crecimiento micelial, germinación y esporulación). El efecto en la inhibición del desarrollo del micelio puede atribuirse primero al ablandamiento de la pared celular, con la posterior interacción del biopolímero (carácter catiónico) con la membrana plasmática, el cual provoca un cambio en la permeabilidad debido a que los grupos amino libres, cargados positivamente en medio ácido, interactúan con los residuos negativos de las macromoléculas expuestas en la pared celular del hongo, afectando también la entrada de nutrientes y afectando su desarrollo micelial (Romanazzi, Feliziani, Baños \& Sivakumar,

\begin{tabular}{|l|c|c|c|c|c|c|}
\hline \multirow{2}{*}{} & \multicolumn{2}{|c|}{$\begin{array}{c}\text { Inhibición del crecimiento } \\
\text { micelial (\%) }\end{array}$} & \multicolumn{2}{c|}{ Germinación (\%) } & \multicolumn{2}{c|}{ Esporulación (esporas/mL) } \\
\cline { 2 - 7 } & C. acutatum & C. gloeosporioides & C. acutatum & C. gloeosporioides & C. acutatum & C. gloeosporioides \\
\hline Control & $0.0 \pm 0.0 \mathrm{a}$ & $0.0 \pm 0.0 \mathrm{a}$ & $100 \pm 0.0 \mathrm{~b}$ & $100 \pm 0.0 \mathrm{~b}$ & $4.11 \times 10^{7} \mathrm{c}$ & $2.65 \times 10^{5} \mathrm{~d}$ \\
\hline $0.10 \%$ & $8.6 \pm 0.07 \mathrm{~b}$ & $6.5 \pm 0.02 \mathrm{~b}$ & $0.0 \pm 0.0 \mathrm{a}$ & $0.0 \pm 0.0 \mathrm{a}$ & $4.00 \times 10^{7} \mathrm{c}$ & $4.02 \times 10^{4} \mathrm{~b}$ \\
\hline $0.50 \%$ & $17.2 \pm 0.23 \mathrm{c}$ & $15.0 \pm 0.01 \mathrm{c}$ & $0.0 \pm 0.0 \mathrm{a}$ & $0.0 \pm 0.0 \mathrm{a}$ & $2.07 \times 10^{7} \mathrm{~b}$ & $2.25 \times 10^{4} \mathrm{a}$ \\
\hline $1.00 \%$ & $17.1 \pm 0.20 \mathrm{c}$ & $21.1 \pm 0.14 \mathrm{~d}$ & $0.0 \pm 0.0 \mathrm{a}$ & $0.0 \pm 0.0 \mathrm{a}$ & $9.50 \times 10^{6} \mathrm{a}$ & $9.50 \times 10^{3} \mathrm{c}$ \\
\hline
\end{tabular}

Se presenta el promedio de 5 réplicas con 3 repeticiones \pm desviación estándar. Letras diferentes indican diferencias significativas entre las concentraciones $(\mathrm{P}<0.05)$.

Tabla I. Efecto del quitosano sobre el desarrollo de $C$. acutatum y $C$. gloeosporioides. 
2017; Verlee, Mincke \& Stevens, 2017; Tovar, ChavesLopez, Serio, Rossi \& Paparella, 2018). Lo anterior fue recientemente reportado por Ramos-Guerrero et al. (2018) al evidenciar por microscopía electrónica de barrido hifas de C. gloeosporioides distorsionadas, presencia de nódulos, adelgazamiento de la pared celular y reducción del diámetro de las hifas expuestas al quitosano (1.0\%) de peso molecular medio. Sin embargo, la efectividad de los resultados de esta investigación son inferiores a lo reportado por RamosGuerrero et al. (2018), debido a que las concentraciones de $1.0 \%$ de quitosano logran una inhibición del $100 \%$ en el desarrollo de C. gloeosporioides aislado de guanábana. En este sentido, existen reportes de baja efectividad del quitosano dependiendo de la cepa evaluada (Hosseinnejad \& Jafari, 2016), como es el caso de Colletotrichum sp. aislado del plátano (Musa sapientum) en el cual sólo se obtuvo un $49 \%$ de inhibición del crecimiento micelial empleando quitosano de medio peso molecular (Gutierrez-Martinez et al., 2018). Para hongos resistentes, el quitosano es incapaz de permeabilizar la membrana celular y permanece en la superficie externa. Palma-Guerrero et al. (2010), mencionan que la resistencia de los hongos al quitosano se debe a que el biopolímero es incapaz de alterar la membrana celular, debido a la composición de fosfolípidos de la membrana. En su estudio demostraron que la disminución en la actividad del quitosano estaba relacionada con una menor cantidad de ácidos grasos insaturados en la membrana celular, por lo tanto la fluidez de la membrana afecta la actividad del quitosano y el mecanismo de acción depende en gran medida de la composición de los hongos. Por otra parte, el proceso de germinación de las esporas requiere de la ruptura de la latencia de la espora, y una serie de procesos que dan lugar a la formación de tubos germinativos, por lo que se requiere de la participación de enzimas (Barhoom \& Sharon, 2004). En este sentido, el quitosano actúa como agente quelante; secuestrando metales esenciales $\left(\mathrm{Mg}^{2+}\right.$ y $\mathrm{Ca}^{2+}$ ), minerales o nutrientes esenciales inhibiendo las reacciones enzimáticas del patógeno (Tovar, Chaves-Lopez, Serio, Rossi \& Paparella, 2018), causando alteraciones en la morfología de las esporas, inhibiendo el proceso de germinación, previamente reportado por Ramos-Guerrero et al.(2018). Para C. gloeosporioides la producción de esporas es de importancia fundamental debido a que permitirá su diseminación y posterior infección (Ansari et al., 2018). Los resultados muestran que para $C$. acutatum se disminuyó la producción de esporas en un rango de $4.00 \times 10^{7}$ a $9.50 \times 10^{6}$ esporas $/ \mathrm{mL}$, en comparación con el control sin tratamiento $\left(4.11 \times 10^{7}\right.$ esporas $\left./ \mathrm{mL}\right)$ y para C. gloeosporioides en un rango de $4.02 \times 10^{4}$ a $9.50 \times 10^{3}$ esporas $/ \mathrm{mL}$ ) comparado con el control sin tratamiento $\left(2.65 \times 10^{5}\right.$ esporas $\left./ \mathrm{mL}\right)$. Los resultados de esta investigación en la esporulación coinciden con lo reportado por Ramos-Guerrero et al. (2018) aplicando quitosano de peso molecular medio al 1.0\%. Estos resultados podrían estar relacionados con la alteración en las estructuras reproductivas inducidos por el quitosano y previamente reportado por Ramos-Guerrero et al. (2018).

Efecto de los aceites esenciales de eucalipto y canela sobre el crecimiento in vitro de $\boldsymbol{C}$. acutatum y $\boldsymbol{C}$. gloeosporioides El crecimiento micelial fue mayormente inhibido mediante la aplicación de aceite esencial de canela (al 2\%) y en el hongo C. gloeosporioides se obtuvo un $50 \%$ de inhibición (Figura 1). Para el caso de C. acutatum los resultados muestran tan solo un $39 \%$ de inhibición. Se observó diferencia significativa $(\mathrm{P}<0.05)$ entre los tratamientos. En este sentido, se ha reportado que la efectividad de los aceites esenciales es mayor cuando se aplican en la fase gaseosa que en la fase líquida, lo que podría explicar la baja inhibición en las cepas evaluadas (Perumal, Sellamuthu, Nambiar \& Sadiku, 2016). La actividad antifúngica de los aceites esenciales depende tanto de las características fisicoquímicas de sus componentes, como de sus grupos funcionales (fenoles, terpenos, alcoholes y cetonas) y del efecto sinérgico

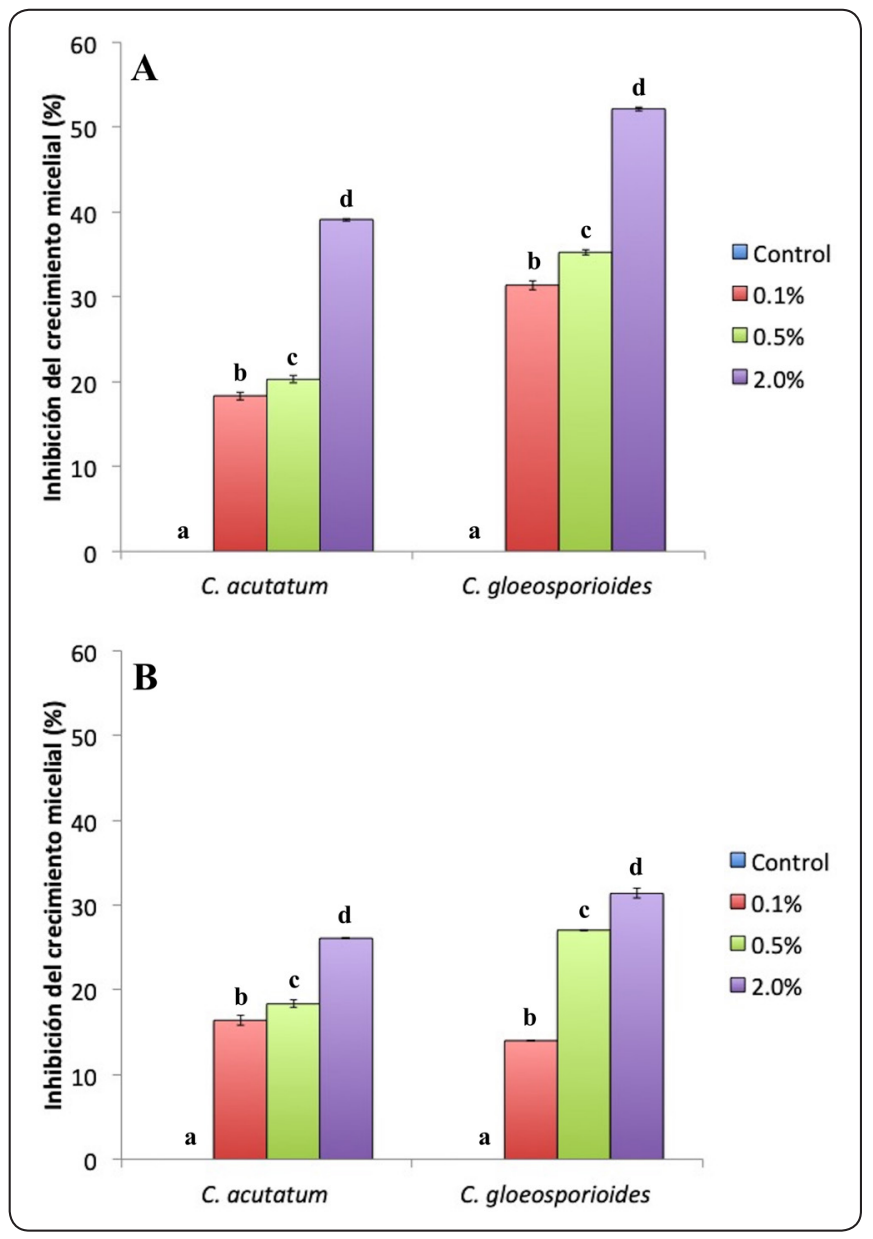

Figura 1. Efecto de los aceites esenciales en el crecimiento micelial. A) canela y B) eucalipto incubado a $25^{\circ} \mathrm{C}$ durante 10 días. Letras diferentes indican diferencias significativas entre los tratamientos $(\mathrm{P}<\mathbf{0 . 0 5})$. 
entre sus componentes (Calo, Crandall, O’Bryan \& Ricke, 2015; Taghavi, Kim \& Rahemi, 2018). En el caso del aceite esencial de canela, está reportado por He et al. (2018) que sus componentes afectan la integridad de la pared y membrana celular de C. acutatum modificando su estructura de manera irreversible, con la consecuente liberación de contenido celular como proteínas solubles, azúcares y ácidos nucleicos; el daño celular es dependiente de la concentración de aceite de canela utilizado, coincidiendo con los resultados de esta investigación. Para C. gloeosporioides se ha reportado que los compuestos lipofílicos en el aceite esencial de canela tienen la capacidad de penetrar y reducir la pared celular, afectando el tamaño de las hifas, causando un daño extensivo en la membrana celular; por otro lado alteran la actividad enzimática afectando la producción de proteínas y de energía (Perumal, Sellamuthu, Nambiar \& Sadiku, 2016; Rabari, Chudashama \& Thaker, 2018). El aceite esencial de eucalipto por su parte mostró una baja capacidad de inhibición en ambas cepas evaluadas, estos resultados coinciden con estudios previos (Combrinck, Regnier \& Kamatou, 2011; España, Arboleda, Ribeiro, Abdelnur \& Guzman, 2017).
Efecto de la combinación de quitosano con aceites esenciales sobre el crecimiento in vitro de $C$. acutatum y $C$. gloeosporioides

La combinación de quitosano $(0.5 \%)$ con aceite esencial de canela fue totalmente efectiva para evitar el crecimiento micelial de C. gloeosporioides y C. acutatum (100\%) (Figura 2 y 3). La germinación fue totalmente inhibida aplicando la mayor concentración de quitosano (Tabla II). Por su parte la esporulación disminuyó con los tratamientos aplicados: Para C. acutatum en un rango de $2.98 \times 10^{7}$ a $2.17 \times 10^{7}$ esporas $/ \mathrm{mL}$ en comparación con el control sin tratamiento $\left(3.23 \times 10^{7}\right.$ esporas $\left./ \mathrm{mL}\right)$ y para $C$. gloeosporioides en un rango de $3.62 \times 10^{4}$ a $3.03 \times 10^{4}$ esporas $/ \mathrm{mL}$ en comparación con el control sin tratamiento $\left(1.91 \times 10^{5}\right.$ esporas $\left./ \mathrm{mL}\right)$ (Tabla II). Se observó diferencia significativa $(\mathrm{P}<0.05)$ entre los tratamientos. Existen reportes que indican que los aceites esenciales alteran la superficie y la estructura de la pared celular de los hongos, por su parte el quitosano puede alterar la síntesis de la pared celular causando liberación de contenido celular; afectando el desarrollo y supervivencia del patógeno (Mohammadi, Hashemi \& Hosseini, 2015).

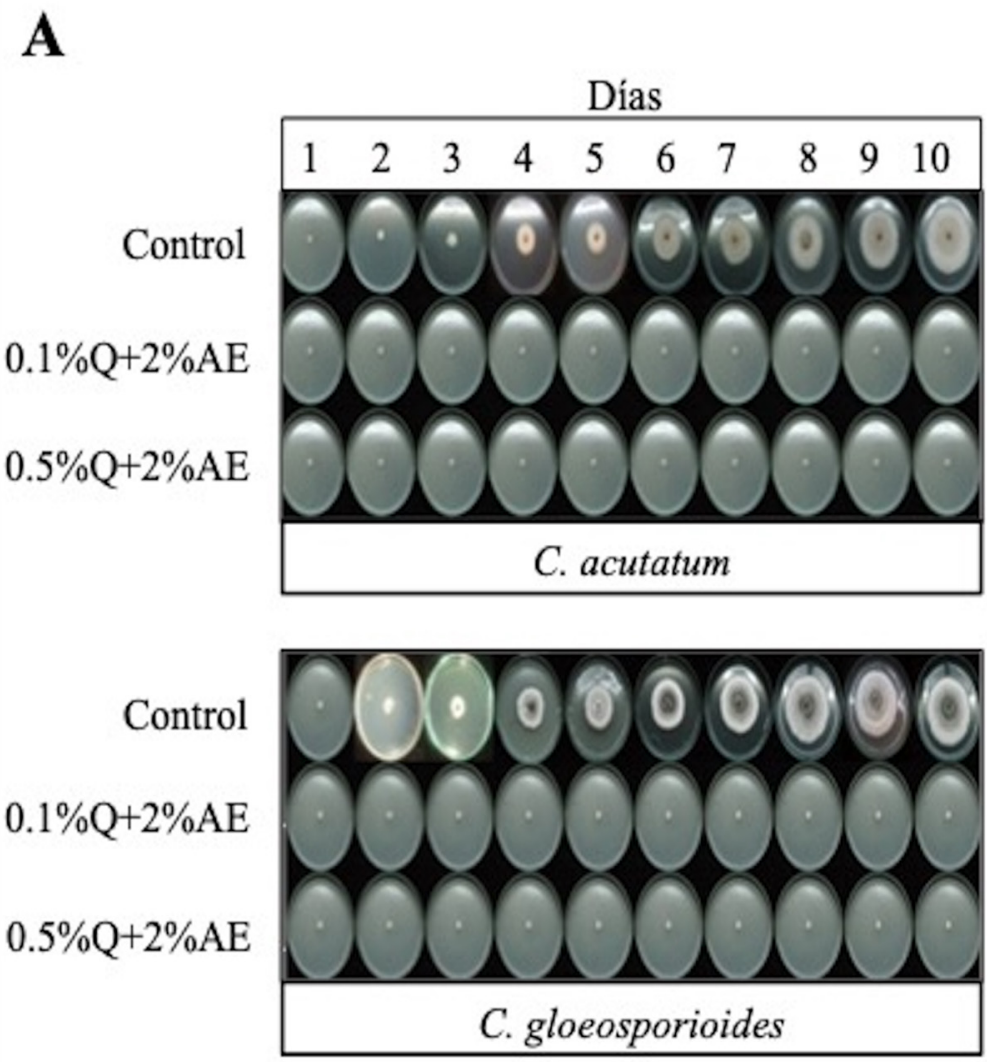

B
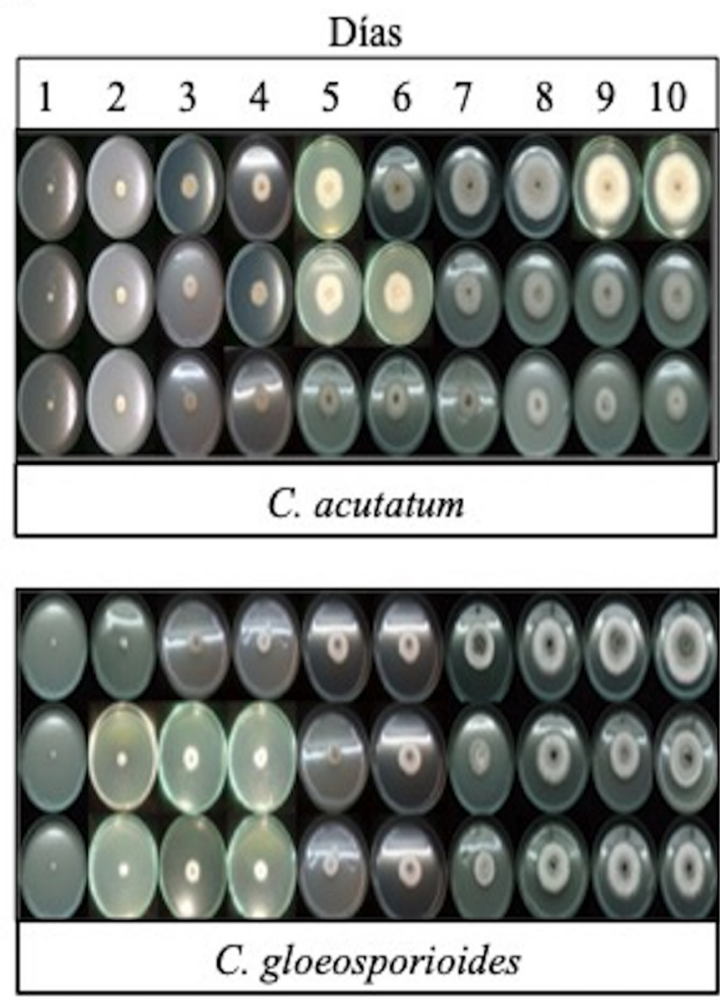

Figura 2. Crecimiento micelial de hongos tratados con quitosano y en combinación con: A) canela y B) eucalipto incubado a $25{ }^{\circ} \mathrm{C}$ durante 10 días. Control: cajas Petri sin tratamientos, Q: quitosano, AE: aceite esencial. 


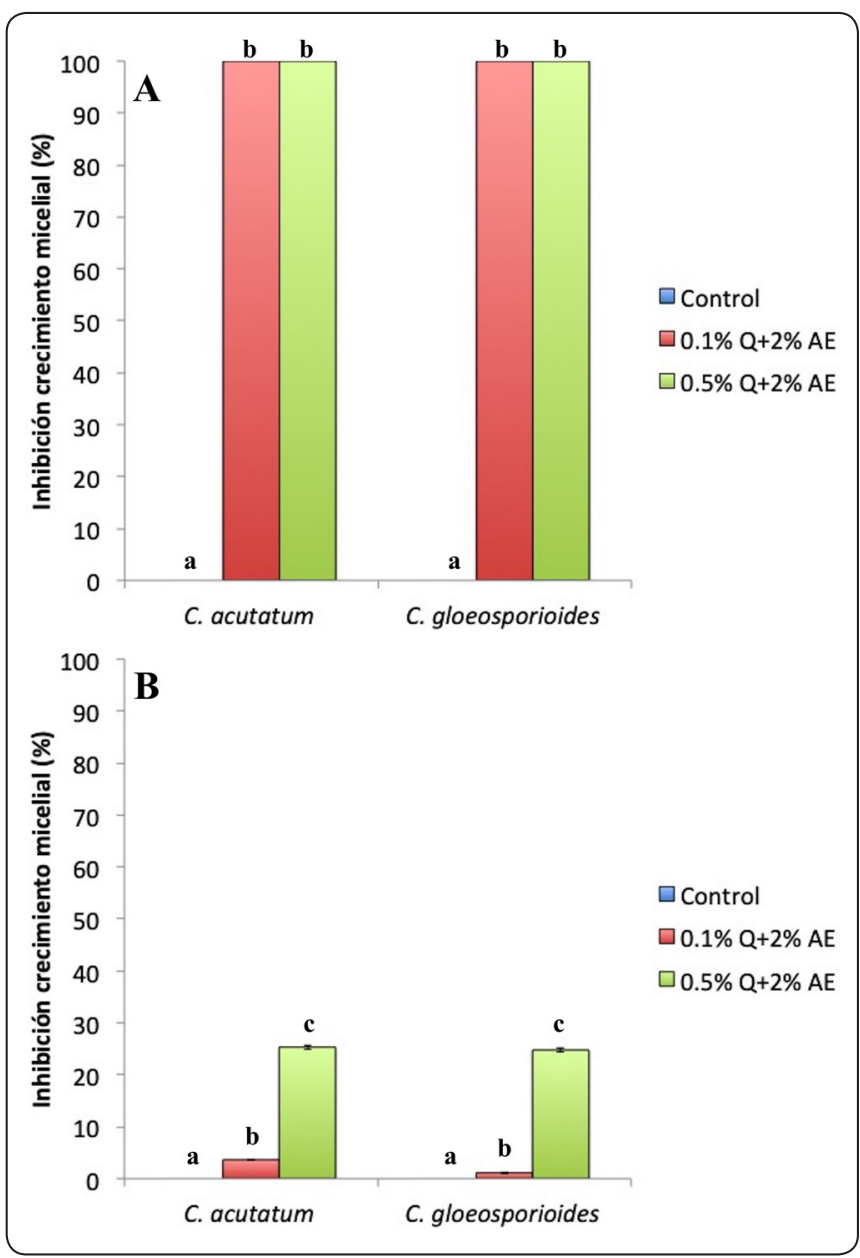

Figura 3. Efecto de la combinación del quitosano con aceites esenciales en la inhibición del crecimiento micelial. A) canela y B) eucalipto incubado a $25^{\circ} \mathrm{C}$ durante 10 días. Letras diferentes indican diferencias significativas entre las concentraciones $(\mathbf{P}<0.05)$.

La efectividad antimicrobiana del quitosano con el aceite esencial de canela ha sido previamente mencionado por (Ojagh, Rezaei, Razavi \& Hosseini, 2010). Los resultados reportados por Ojagh, Rezaei, Razavi \& Hosseini (2010) coindicen con lo citado en esta investigación, al encontrar que la sola aplicación de quitosano no es suficiente para el control de Listeria monocytogenes, Escherichia coli, Lactobacillus plantarum, Lactobacillus sakei y Pseudomonas fluorescens; debido a que el control microbiano efectivo se logró con la combinación del biopolímero y el aceite esencial de canela (al 2\%). En este estudio, además se demostró que el aceite esencial de canela incorporado en la matriz polimérica del quitosano es capaz de difundirse, lo cual es promisorio considerando su potencial aplicación en frutos para el control de enfermedades. Sin embargo, aun cuando la actividad antimicrobiana del quitosano puede mejorarse con la formación de complejos con otros aditivos, con la aplicación del quitosano $(0.5 \%)$ y el aceite esencial de eucalipto (2\%) tan solo se obtuvo un $24 \%$ de inhibición del crecimiento micelial (Figura 3). En este sentido, en investigaciones previas se ha mencionado que la eficacia de la actividad antifúngica del quitosano en combinación con aceites esenciales depende no sólo de la capacidad de liberación del aceite esencial de la matriz del biopolímero y la cepa evaluada, sino también del aceite esencial utilizado con respecto a su polaridad, estructura química y la formación de entrecruzamientos entre moléculas (Mohammadi, Hashemi \& Hosseini, 2015; Tovar, Chaves-Lopez, Serio, Rossi \& Paparella, 2018). Los resultados sugieren que la combinación del quitosano con el aceite esencial de eucalipto afecta la eficacia tanto del quitosano como del aceite esencial.

\section{Conclusiones}

Los resultados son importantes por el daño que un crecimiento eficiente de las hifas desempeña en la colonización fúngica de los tejidos de las plantas. Por otro lado, la esporulación es un evento crucial para el desarrollo de hongos, por lo que una inhibición satisfactoria en este proceso puede romper los ciclos de infección en las frutas susceptibles. Finalmente, los tratamientos efectivos para controlar el proceso de germinación son de gran valor porque a las esporas se les reconoce como el inóculo que propaga la enfermedad, por lo tanto, la aplicación combinada del quitosano con aceite esencial de canela puede ser una estrategia para la preservación de frutos de aguacate y como una alternativa al uso de fungicidas en el control de la antracnosis.

\begin{tabular}{|l|c|c|c|c|}
\hline & \multicolumn{2}{|c|}{ Germinación (\%) } & \multicolumn{2}{c|}{ Esporulación (esporas/mL) } \\
\hline & C. acutatum & C. gloeosporioides & C. acutatum & C. gloeosporioides \\
\hline Control & $100 \pm 0.0 \mathrm{c}$ & $100 \pm 0.0 \mathrm{~b}$ & $3.23 \times 10^{7} \mathrm{c}$ & $1.91 \times 10^{5} \mathrm{c}$ \\
\hline $0.1 \% \mathrm{Q}+2 \% \mathrm{AE}$ & $10 \pm 0.0 \mathrm{~b}$ & $0.0 \pm 0.0 \mathrm{a}$ & $2.98 \times 10^{7} \mathrm{~b}$ & $3.62 \times 10^{4} \mathrm{~b}$ \\
\hline $0.5 \% \mathrm{Q}+2 \% \mathrm{AE}$ & $0.0 \pm 0.0 \mathrm{a}$ & $0.0 \pm 0.0 \mathrm{a}$ & $2.17 \times 10^{7} \mathrm{a}$ & $3.03 \times 10^{4} \mathrm{a}$ \\
\hline
\end{tabular}

Se presenta el promedio de 5 réplicas con 3 repeticiones \pm desviación estándar. Letras diferentes indican diferencias significativas entre las concentraciones $(\mathrm{P}<0.05)$.

Tabla II. Efecto del quitosano en combinación con aceite esencial de canela sobre el desarrollo de C. acutatum y C. gloeosporioides. 


\section{Agradecimientos}

Al Tecnológico Nacional de México (TecNM) por las facilidades otorgadas para la realización de este proyecto de investigación.

\section{REFERENCIAS}

Ansari, A., Khanzada, M. A., Rajput, M. A., Maitlo, S., Qayoom, A. \& Rajput, A. U. (2018). Effect of different abiotic factors on the growth and sporulation of Colletotrichum gloeosporioides causing anthracnose of mango. Plant Protection, 2(01), 23-30.

Barhoom, S. \& Sharon, A. (2004). cAMP regulation of "pathogenic" and "saprophytic" fungal spore germination. Fungal Genetics and Biology, 41, 317-326.https://doi. org/10.1016/j.fgb.2003.11.011.

Calo, J. R., Crandall, P. G., O'Bryan, C. A. \& Ricke, S. C. (2015). Essential oils as antimicrobials in food systems-A review. Food Control, 54, 111-119.https://doi. org/10.1016/j.foodcont.2014.12.040.

Combrinck, S., Regnier, T. \& Kamatou, G. P. P. (2011). In vitro activity of eighteen essential oils and some major components against common postharvest fungal pathogens of fruit. Industrial Crops and Products, 33(2), 344-349. https://doi.org/10.1016/j.indcrop.2010.11.011.

España, M. D., Arboleda, J. W., Ribeiro, J. A., Abdelnur, P. V. \& Guzman, J. D. (2017). Eucalyptus leaf byproduct inhibits the anthracnose-causing fungus Colletotrichum gloeosporioides. Industrial Crops and Products, 108, 793-797. https://doi.org/10.1016/j.indcrop.2017.08.002.

Fagundes, C., Pérez-Gago, M. B., Monteiro, A. R. \& Palou, L. (2013). Antifungal activity of food additives in vitro and as ingredients of hydroxypropyl methylcellulose-lipid edible coatings against Botrytis cinerea and Alternaria alternata on cherry tomato fruit. International Journal of Food Microbiology, 166(3), 391-398. https://doi. org/10.1016/j.ijfoodmicro.2013.08.001.

El Ghaouth, A., Arul, J., Grenier, J. \& Asselin, A. (1992). Antifungal activity of chitosan on two postharvest pathogens of strawberry fruits. Phytopathology, 82(4), 398-402.

González-Estrada, R. R., Calderón-Santoyo, M., RagazzoSánchez, J. A., Peyron, S. \& Chalier, P. (2018). Antimicrobial soy protein isolate based films: physical characterization, active agent retention and antifungal properties against Penicillium italicum. International Journal of Food Science \& Technology, 53(4), 921-929. https://doi.org/10.1111/ijfs.13664.

Gutierrez-Martinez, P., Ledezma-Morales, A., del Carmen Romero-Islas, L., Ramos-Guerrero, A., Romero-Islas, J., Rodríguez-Pereida, C. \& González-Estrada, R. (2018). Antifungal Activity of Chitosan against Postharvest Fungi of Tropical and Subtropical Fruits. In Chitin-ChitosanMyriad Functionalities in Science and Technology.
IntechOpen.https://doi: 10.5772/intechopen.76095.

He, J., Qin, W., Wu, D., Zhang, Q., Chen, H., Li, H. \& Yuan, J. (2018). Efficacy and mechanism of cinnamon essential oil on inhibition of Colletotrichum acutatum isolated from 'Hongyang'kiwifruit. Frontiers in Microbiology, 9, 1288.https://doi.org/10.3389/fmicb.2018.01288.

Hosseinnejad, M. \& Jafari, S. M. (2016). Evaluation of different factors affecting antimicrobial properties of chitosan. International Journal of Biological Macromolecules, 85, 467-475. https://doi.org/10.1016/j. ijbiomac.2016.01.0224.

Kim, K. H., Kabir, E. \& Jahan, S. A. (2017). Exposure to pesticides and the associated human health effects. Science of the Total Environment, 575, 525-535. https:// doi.org/10.1016/j.scitotenv.2016.09.009.

Mohammadi, A., Hashemi, M. \& Hosseini, S. M. (2015). Nanoencapsulation of Zataria multiflora essential oil preparation and characterization with enhanced antifungal activity for controlling Botrytis cinerea, the causal agent of gray mould disease. Innovative Food Science \& Emerging Technologies, 28, 73-80. https://doi. org/10.1016/j.ifset.2014.12.011.

Ojagh, S. M., Rezaei, M., Razavi, S. H. \& Hosseini, S. M. H. (2010). Development and evaluation of a novel biodegradable film made from chitosan and cinnamon essential oil with low affinity toward water. Food Chemistry, 122(1), 161-166.https://doi.org/10.1016/j. foodchem.2010.02.033.

Palma-Guerrero, J., Lopez-Jimenez, J. A., Pérez-Berná, A. J., Huang, I. C., Jansson, H. B., Salinas, J.\& Lopez-Llorca, L. V. (2010). Membrane fluidity determines sensitivity of filamentous fungi to chitosan. Molecular Microbiology, 75(4), 1021-1032. https://doi.org/10.1111/j.1365-2958. 2009.07039.x.

Perumal, A. B., Sellamuthu, P. S., Nambiar, R. B. \& Sadiku, E. R. (2016). Antifungal activity of five different essential oils in vapour phase for the control of Colletotrichum gloeosporioides and Lasiodiplodia theobromae in vitro and on mango. International Journal of Food Science \& Technology, 51(2), 411-418. https://doi.org/10.1111/ ijfs. 12991.

Prakash, B., Kedia, A., Mishra, P. K. \& Dubey, N. K. (2015). Plant essential oils as food preservatives to control moulds, mycotoxin contamination and oxidative deterioration of agri-food commodities-Potentials and challenges. Food Control, 47, 381-391. https://doi.org/10.1016/j. foodcont.2014.07.023.

Rabari, V. P., Chudashama, K. S. \& Thaker, V. S. (2018). In vitro Screening of 75 Essential Oils Against Colletotrichum gloeosporioides: A Causal Agent of Anthracnose Disease of Mango. International Journal of Fruit Science, 18(1), 1-13https://doi.org/10.1080/15538362.2017.1377666.

Ramos-Guerrero, A., González-Estrada, R. R., Hanako-Rosas, G., Bautista-Baños, S., Acevedo-Hernández, G., Tiznado- 
Hernández, M. E. \& Gutiérrez-Martínez, P. (2018). Use of inductors in the control of Colletotrichum gloeosporioides and Rhizopus stolonifer isolated from soursop fruits: in vitro tests. Food Science and Biotechnology, 27(3), 755763. https://doi.org/10.1007/s10068-018-0305-5.

Reganold, J. P. \& Wachter, J. M. (2016). Organic agriculture in the twenty-first century. Nature Plants, 2(2), 15221. http://dx.doi.org/10.1038/nplants.2015.221.

Romanazzi, G., Feliziani, E., Baños, S. B. \& Sivakumar, D. (2017). Shelf life extension of fresh fruit and vegetables by chitosan treatment. Critical Reviews in Food Science and Nutrition, 57(3), 579-601.https://doi.org/10.1080/10 408398.2014.900474.

SAGARPA (2017). Planeación agrícola nacional 20172030. Available at: https://www.gob.mx/cms/uploads/ attachment/file/257067/Potencial-Aguacate.pdf (Accessed: 12 October 2018).

Sarkhosh, A., Vargas, A. I., Schaffer, B., Palmateer, A. J., Lopez, P., Soleymani, A. \& Farzaneh, M. (2017). Postharvest management of anthracnose in avocado (Persea americana Mill.) fruit with plant-extracted oils. Food Packaging and Shelf Life, 12, 16-22. https://doi. org/10.1016/j.fpsl.2017.02.001.

Taghavi, T., Kim, C. \& Rahemi, A. (2018). Role of Natural Volatiles and Essential Oils in Extending Shelf Life and
Controlling Postharvest Microorganisms of Small Fruits. Microorganisms, 6(4), 104. https://doi.org/10.3390/ microorganisms6040104.

Tovar, C. D. G., Chaves-Lopez, C., Serio, A., Rossi, C. \& Paparella, A. (2018). Chitosan coatings enriched with essential oils: Effects on fungal decay of fruits and mechanisms of action. Trends in Food Science \& Technology, 78, 61-71.https://doi.org/10.1016/j. tifs.2018.05.019.

Trinidad-Ángel, E., Ascencio-Valle, F. D. J., Ulloa, J. A., Ramírez-Ramírez, J. C., Ragazzo-Sánchez, J. A., Calderón-Santoyo, M. \& Bautista Rosales, P. U. (2017). Identificación y caracterización de Colletotrichum spp. causante de antracnosis en aguacate Nayarit, México. Revista Mexicana de Ciencias Agrícolas, 8(SPE19), 39533964.http://dx.doi.org/10.29312/remexca.v0i19.664.

Verlee, A., Mincke, S. \& Stevens, C. V. (2017). Recent developments in antibacterial and antifungal chitosan and its derivatives. Carbohydrate Polymers, 164, 268-283. https://doi.org/10.1016/j.carbpol.2017.02.001.

Yao, H., Tian, S. \& Wang, Y. (2004). Sodium bicarbonate enhances biocontrol efficacy of yeasts on fungal spoilage of pears. International Journal of Food Microbiology, 93(3), 297-304.https://doi.org/10.1016/j. ijfoodmicro.2003.11.011. 